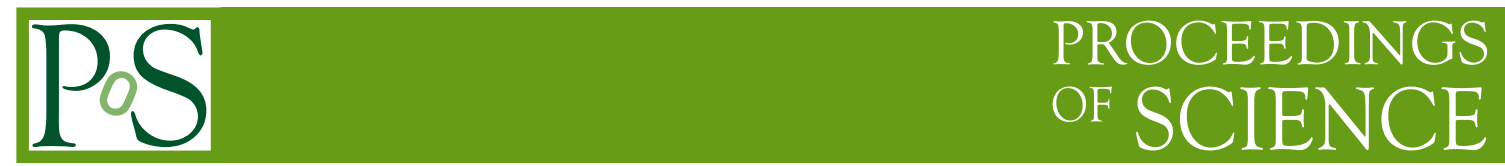

\title{
Tevatron top physics
}

\section{Jeannine WAGNER-KUHR*}

on behalf of the CDF and DØ Collaborations

University of Karlsruhe, Karlsruhe, Germany

E-mail: jwagnereekp.uni-karlsruhe.de

A summary of the most recent results on top quark physics obtained at Fermilab's Tevatron proton-antiproton collider, operating at a centre of mass energy of $1.96 \mathrm{TeV}$, is presented. Measurements of the top pair and single top quark production cross sections, the investigation of top quark decay properties, the precision measurement of the top quark mass as well as searches for physics beyond the standard model are discussed.

Physics at LHC 2008

29 September - October 4, 2008

Split, Croatia

${ }^{*}$ Speaker. 


\section{Introduction}

In 1995 the top quark was discovered at the Tevatron proton-antiproton collider at Fermilab by the $\mathrm{CDF}$ and $\mathrm{D} \emptyset$ collaborations [1]. It is the most massive known elementary particle and its mass of the order of the electroweak symmetry breaking scale suggest that it may play a special role in new physics models. So far the Tevatron collider is the only place to study the top quark.

At the Tevatron collider, with a center of mass energy of $\sqrt{s}=1.96 \mathrm{TeV}$, most top quarks are pair produced via the strong interaction. About $85 \%$ of the top pairs are produced via quark-antiquark annihilation and $15 \%$ via gluon fusion. Beside the production of top quarks in pairs also the production of single top quarks in electroweak interaction should exist and its cross section is predicted to be $\sim 2.5$ smaller than the top pair production cross section.

In the standard model the top quark decays predominantly into a $W$ boson and a $b$ quark, with a branching ratio close to $100 \%$. Different decay channels are distinguished according to the decay mode of the $W$ boson.

Top pair events with both $W$-bosons decaying leptonically to $e v$ or $\mu \nu$ are called dilepton events, events with only one $W$-boson decaying leptonically are called lepton+jets events and events with both $W$-bosons decaying hadronically are called all hadronic events. Most of the top pair measurements are carried out in the lepton+jets channel, which is characterized by moderate background and a branching ratio of about $30 \%$. In single top quark events we consider only the leptonic decay of the $W$-boson, the branching ratio is here about $21 \%$.

\section{Top Quark Properties in Production and Decay}

Investigating top quark properties in production and decay will answer the question whether the observed top quark behaves actually like the top quark predicted in the standard model (SM). In this article the production cross section for top pairs and single top quarks, characteristics of top pair production like the fraction of gluon fusion production and the forward-backward charge asymmetry, and the Wtb coupling are discussed.

\subsection{Single Top Quark Production Cross Section}

The challenge of measuring the single top quark cross section at the Tevatron is the huge background of $\mathrm{W}+\mathrm{jets}$ events. A simple cut based event selection is here not sufficient and to improve the sensitivity both experiments use multivariate techniques, like likelihood functions, decision trees, matrix element methods and neural networks. An overview of the most recent single top quark cross section measurements performed at the Tevatron is presented in figure 1. The CDF likelihood function, neural network and matrix element discriminants have been combined in a neural network to obtain a super discriminant. An analysis employing this technique on $2.2 \mathrm{fb}^{-1}$ saw an excess of signal over background, and a cross section of $2.2_{-0.6}^{+0.7} \mathrm{pb}$ is obtained [2]. The result is below but consistent with the SM prediction of $2.9 \pm 0.4 \mathrm{pb}$. The expected signal significance of the CDF combination is $5.1 \sigma$ while a significance of $3.7 \sigma$ is observed. The three multivariate analyses [ [ $]$ ] from $\mathrm{D} \varnothing$ have been combined using BLUE, the best linear unbiased estimator. A cross section of $4.7 \pm 1.3 \mathrm{pb}$ is obtained, which is larger than but consistent with the SM prediction. The expected signal significance of the $\mathrm{D} \emptyset$ combination is $2.3 \sigma$ while a significance of $3.6 \sigma$ has 

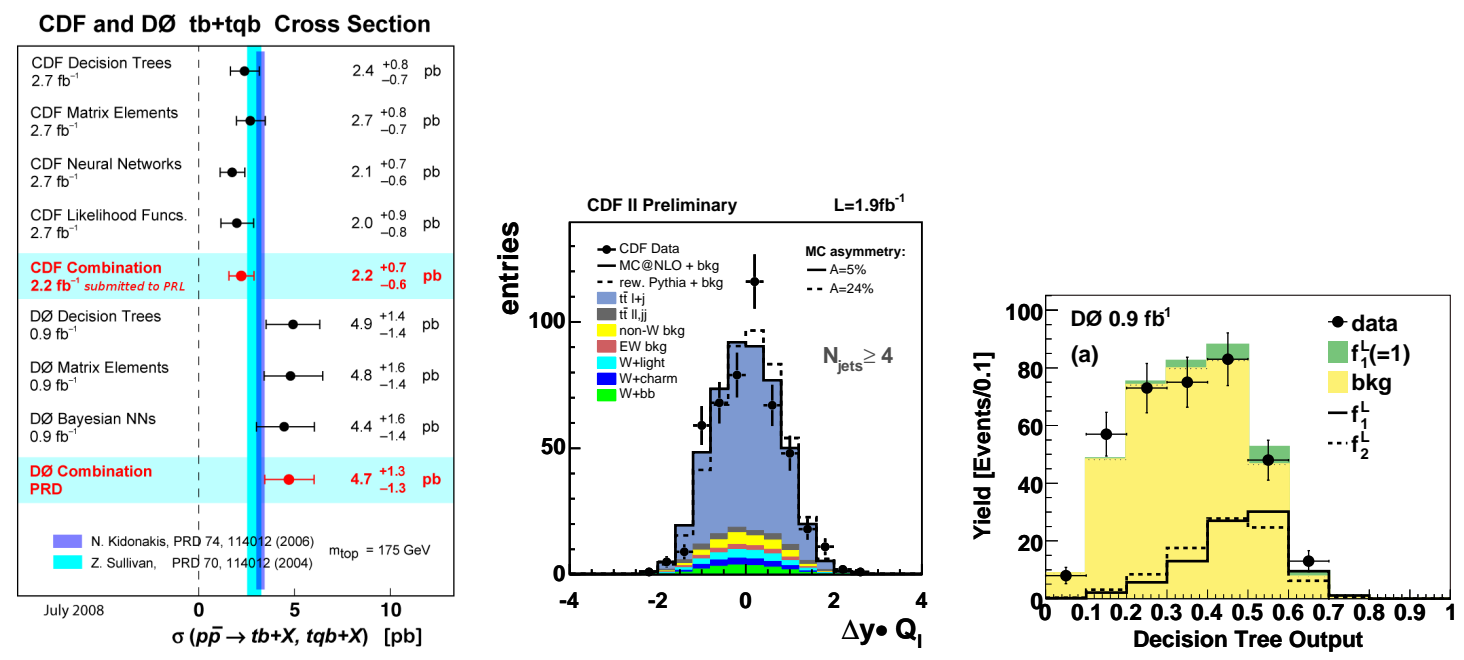

Figure 1: Left: Single top quark production. Shown is an overview of the cross section measurements performed at the Tevatron. Middle: Measurement of the forward-backward charge asymmetry. Shown is the reconstructed rapidity difference of the top and anti-top quark. Right: Study of the Wtb coupling. Shown is the single top quark boosted decision tree discriminant assuming $f_{1}^{R}=f_{2}^{R}=0$.

been observed. Both Tevatron experiments see strong evidence for electroweak single top quark production.

\subsection{Top Pair Production}

The top pair production cross section has been measured in different decay channels and with different methods. In contrast to the single top quark production measurement, many top pair cross section measurements are counting experiments, as long as they are performed in regions with low background (e.g. lepton+jets channel with 4 jets and at least one jet tagged as $b$-jet). The most precise measurements are obtained in the lepton+jets channel. All top pair cross section measurements are consistent with each other and assuming a top quark mass of $175 \mathrm{GeV} / \mathrm{c}^{2}$ the combination of the CDF measurements yields a cross section of $7.0 \pm 0.3$ (stat.) \pm 0.4 (syst.) \pm 0.4 (lumi) pb, while the combination of the $\mathrm{D} \emptyset$ results yields $7.8 \pm 0.5$ (stat.) \pm 0.6 (syst.) \pm 0.5 (lumi) pb [ [П]. Thus, the top pair production cross section is now known with a relative uncertainty of about $9 \%$ at Tevatron center of mass energies.

The fraction of top pair events produced via gluon fusion has been measured in three CDF analyses [5]. Two analyses use variables which are sensitive to the different spin configuration of the top quarks in gluon fusion and quark-antiquark annihilation events. The analysis performed in the lepton+jets uses a neural network while the more recent analysis in the dilepton channel utilizes just the azimuthal angle difference of the two charged leptons. A third analysis, performed in the lepton+jets channel, exploits the fact that gluons emit more soft gluons than quarks and so the number of tracks with small transverse momentum is used as sensitive variable. The combination of both lepton+jets analyses yields a gluon fusion fraction of $\left(7_{-7}^{+15}\right) \%\left(1 \mathrm{fb}^{-1}\right)$, while the dilepton analysis obtains a fraction of $\left(53_{-38}^{+36}\right) \%\left(2 \mathrm{fb}^{-1}\right)$. Both results are consistent with the SM prediction of about $15 \%$. 
At the Tevatron a small forward-backward asymmetry of about 5\% is predicted in SM top pair production. In new physics scenarios with a $Z^{\prime}$ or an axigluon this asymmetry can be as large as $\pm 30 \%$. The asymmetry is frame dependent and is predicted to be largest in the top pair rest frame. To measure the asymmetry the lepton+jets channel is used and the asymmetry in the top pair rest frame has been studied by both experiments, using the rapidity difference of the top and anti-top. This quantity is presented in figure 1 for the CDF analysis. The CDF collaboration performed also an asymmetry measurement in the laboratory frame using the polar angle of the hadronically decaying top quark as sensitive variable. The $\mathrm{D} \emptyset$ collaboration obtains an uncorrected asymmetry of $(12 \pm 8) \%$ [6], while an asymmetry of $0.8 \%$ is predicted. To account for sizable acceptance and measurement dilution effects the CDF collaboration corrects the raw results for these effects leading to an asymmetry of $(24 \pm 14) \%$ in the top rest frame and to $(18 \pm 8) \%$ in the laboratory frame [7]. The measured asymmetries at the Tevatron are larger but consistent with the SM prediction within uncertainties.

\subsection{Wtb coupling}

The general $W t b$ vertex contains besides the left-handed vector coupling $f_{1}^{L}$ also a right-handed vector coupling $f_{1}^{R}$ and tensor couplings $f_{2}^{L}$ and $f_{2}^{R}$. In the SM only $f_{1}^{L}$ is non-zero, namely one, and non-SM couplings would affect the single top quark production in rate and kinematic distributions. The $\mathrm{D} \emptyset$ collaboration uses the single top quark boosted decision tree discriminant to determine two couplings at a time while the other two are assumed to be negligible, see figure 1. This analysis yields for the scenario with $f_{1}^{R}=f_{2}^{R}=0$ the couplings $f_{1}^{L}=1.4_{-0.5}^{+0.6}$ and $f_{2}^{L}<0.5$ at 95\% C.L. [8]. Besides single top quark production also the helicity of $W$-bosons in top pair decays would be affected by non-SM $W t b$ couplings. In the SM the fraction of longitudinally polarized $W$ bosons is $f_{0}=0.7$, the fraction of left-handed $W$-bosons $f_{-}=0.3$ and the fraction of right-handed W-bosons is $f_{+}=0.0$. The reconstructed $\cos \theta^{*}$, with $\theta^{*}$ being the angle between the charged lepton or the down type quark and the negative direction of the top quark in the rest frame of the $\mathrm{W}$ boson, is here used as sensitive variable. The most recent CDF analyses use the leptonically decaying $W$ boson in the lepton+jet channel and two analyses have been combined with BLUE. The DØ analysis uses also the dilepton channel and uses both $W$ bosons in each event in case of the lepton+jets channel. The reconstructed $\cos \theta^{*}$ distribution is fitted and the CDF combination yields for the simultaneous fit $0.66 \pm 0.16$ for $f_{0}$ and $-0.03 \pm 0.07$ for $f_{+}$while the $\mathrm{D} \varnothing$ collaboration obtains $0.49 \pm 0.13$ for $f_{0}$ and $0.11 \pm 0.07$ for $f_{+}$[9]. All $W$-helicity measurements in top pair events performed at the Tevatron are compatible with the SM prediction.

\section{Top Mass Measurement}

The mass $m_{t}$ of the top quark is an important standard model parameter and precision measurements of the top quark and $W$ masses test the consistency of the SM, and in particular the Higgs mechanism. In order to measure the top quark mass top pair events in all decay channels are used and to reduce the systematic uncertainty due to the jet energy scale uncertainty the jet energy scale (JES) is calibrated in an in situ measurement using the hadronically decaying $W$ boson in the lepton+jets and all hadronic channel. The CDF collaboration has also performed measurements in the lepton+jets channel using not the reconstructed top mass as sensitive variable but the transverse 

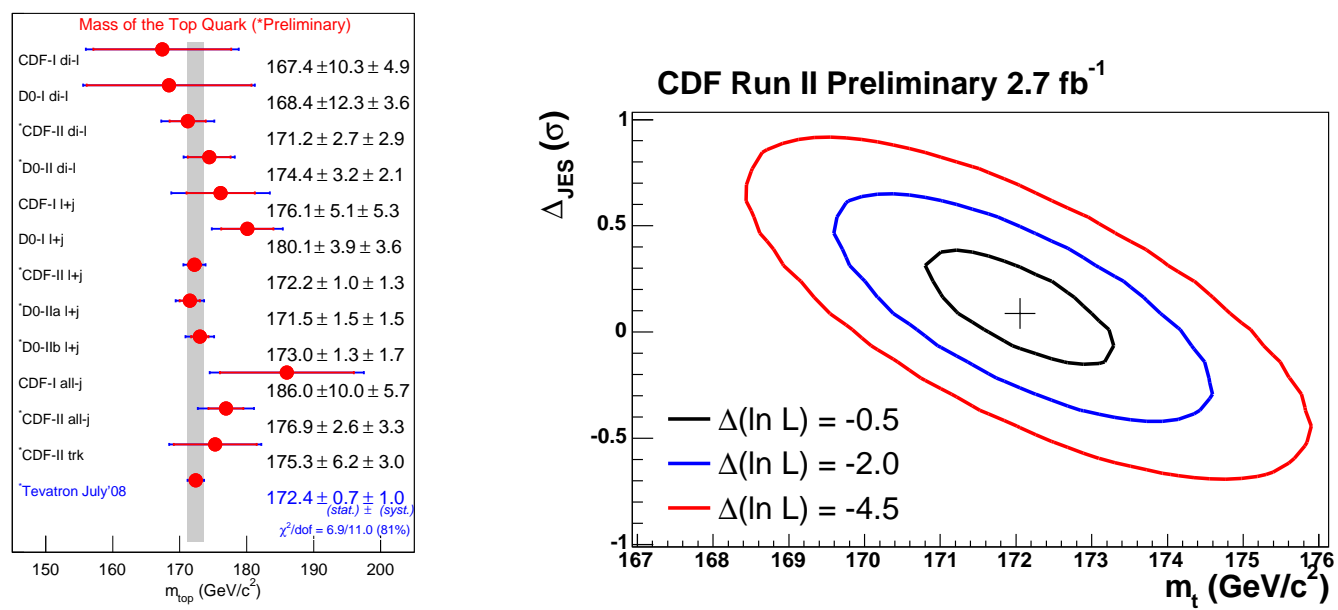

Figure 2: Measurement of the top quark mass. Left: Tevatron top mass combination. Right: Most precise top mass measurement in the lepton+jets channel performed by the CDF collaboration.

momentum of the charged lepton and the decay length of $b$-tagged jets. These variables are almost entirely independent of the jet energy scale and thus provide a cross check on the measurements involving JES, albeit statistically limited. The combination of all these measurements, see figure 2, yields a top mass of $172.4 \pm 0.7$ (stat.) \pm 1.0 (syst.) $\mathrm{GeV} / \mathrm{c}^{2}$ [10], meaning that the top quark mass is now known with a relative precision of $0.7 \%$. The measurements performed in all channels and with different methods are consistent with each other.

The most precise top quark mass measurements are carried out in the lepton+jets channel and the most recent lepton+jets measurements use the matrix element method, the in situ jet energy scale calibration and neural networks. Figure $\mathrm{Q}$ shows for the CDF measurement the likelihood contours as a function of the top mass and the in situ jet energy scale parameter. The most precise single measurements of both experiments yield a top mass of $172.2 \mathrm{GeV} / \mathrm{c}^{2}$ with a relative uncertainty of about $1 \%$ [11].

\section{Search for New Physics in the Top Quark Sector}

Since the top quark is the only known fermion with a mass of the order of the electroweak symmetry breaking scale it may play a special role in new physics models. Searches for new physics in top quark events and in events with top quark signature (e.g. lepton+jets channel) are discussed.

Both Tevatron experiments performed searches for heavy massive resonances in top pair [12] and single top quark production [13]. In case of top pair events the reconstructed mass of the top quark pair system is studied and $D \emptyset$ and CDF find so far no hint for a heavy resonance, e.g. $Z^{\prime}$, heavy gluon. The $\mathrm{D} \emptyset$ collaboration sets for example a lower limit on a narrow leptophobic $Z^{\prime}$ of $760 \mathrm{GeV} / \mathrm{c}^{2}$. To search for resonant single top quark production (e.g. charged Higgs, $W^{\prime}$ ) the invariant mass of the reconstructed top quark and the additional $b$-jet is used. Both Tevatron experiments find so far no hints for massive resonances in single top quark production. 

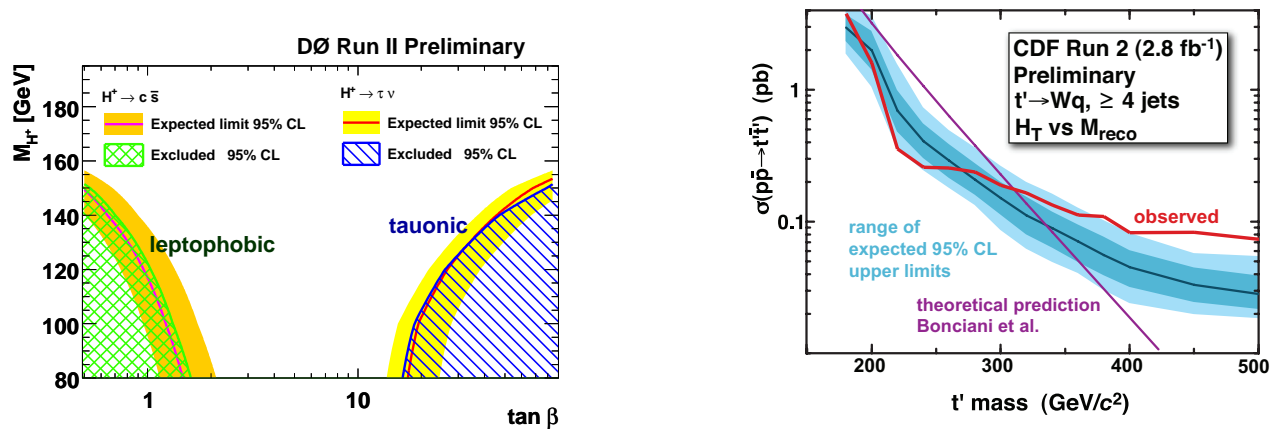

Figure 3: Search for new physics in the top quark sector. Left: Search for a charged Higgs in the decay of top pair events. Shown is the limit as a function of the charged Higgs mass and $\tan \beta$ obtained by the D $\varnothing$ analysis. Right: Search for $t^{\prime}$ pair production. Shown is the limit on the $t^{\prime}$ production cross section as a function of the reconstructed $t^{\prime}$ mass.

The CDF and DØ collaboration have also performed searches for a charged Higgs in the decay of top pair events [14]. The decay $t \rightarrow b H^{+}$is allowed in the minimal super-symmetric SM (MSSM). At low $\tan \beta$ the decay $H^{+} \rightarrow c \bar{s}$ is important while at large $\tan \beta$ the decay $H^{+} \rightarrow \tau v$ becomes dominant. Here $\tan \beta$ is the ratio of the vacuum expectation values of the two Higgs doublets. The CDF collaboration searches for $H^{+} \rightarrow c \bar{s}$ by using the invariant mass of the two light-quark jets assigned as jets originating from the charged Higgs. The DØ collaboration chose a different ansatz and searches for a charged Higgs decaying into either a $c \bar{s}$ or $\tau \nu$ by measuring the rate of top pair events across the decay channels: lepton+jets, dilepton and tau+lepton. The limits as a function of the charged Higgs mass and $\tan \beta$ obtained by the $D \varnothing$ analysis are presented in figure 3 .

Most recent searches for flavor changing neutral currents (FCNC) have been performed by the CDF collaboration. In top pair events CDF searches for FCNC decays like $t \rightarrow Z q$ by looking at the $\chi^{2}$ of a kinematic fit with FCNC hypothesis. From a fit to this $\chi^{2}$ variable an upper limit of $3.7 \%$ for the branching ratio $t \rightarrow Z c$ is derived [15]. The CDF collaboration searches also for FCNC in single top quark production and looks here for events where the fusion of a gluon and a $u$ - or $c$-quark leads to a single top quark. A neural network is used to separate FCNC top quark events from background events. From a fit to the neural network output an upper limit on the anomalous single top quark cross section of $1.8 \mathrm{pb}$ is determined and upper limits on the anomalous couplings of $\kappa_{g t u}=0.018 \mathrm{TeV}^{-1}$ and $\kappa_{g t c}=0.069 \mathrm{TeV}^{-1}$ are derived [16].

Searches for new physics in events with top quark signature have been performed by both Tevatron experiments. Here, only the most recent results are discussed. The CDF collaboration searches in events with top pair lepton+jets signature for a heavy top like quark [17], a $t^{\prime}$, decaying into a $W$ boson and a quark $(b, s, d)$ assuming that the mass split between a $b^{\prime}$ and a $t^{\prime}$ is below the $W$ boson mass. The reconstructed $t^{\prime}$ mass as well as the total transverse energy in the event are used in a 2-dimensional likelihood fit leading to the limit on the $t^{\prime}$ production cross section presented in figure 3. In events with top pair dilepton signature CDF searches for a light super-symmetric top partner $\left(\lesssim m_{t}\right)$, the stop [18]. In this analysis, it is assumed that the stop decays with $100 \%$ into a $b$ quark and a chargino which would then decay via different modes into a neutralino, a 
charged lepton, and a neutrino. The stop mass is reconstructed for each event assuming the pair of neutralino and neutrino to be a pseudo particle and limits are extracted from this variable showing that there is so far no hint for a stop in the data.

\section{Conclusion}

In this article the most recent results on top quark physics from the Tevatron have been presented. Many efforts have been made to study the top quark and so far the top quark behaves as predicted in the standard model. The top quark mass is measured very precisely with a relative precision of $0.7 \%$ and so far no significant hints for new physics in top quark events as well as in events with top quark signature have been found. Most measurements of top quark properties are limited by statistics, so we expect these to continue to improve in precision along with the sensitivity to new physics as more data is collected.

\section{References}

[1] F. Abe et al. (CDF Collaboration), Phys. Rev. Lett. 74, 2626 (1995); S. Abachi et al. (DØ Collaboration), Phys. Rev. Lett. 74, 2632 (1995).

[2] T. Aaltonen et al. (CDF Collaboration), Phys. Rev. Lett. 101, 252001 (2008); public CDF notes 9479, 9451, 9464, 9445.

[3] V.M. Abazov et al. (DØ Collaboration), Phys. Rev. D78, 012005 (2008).

[4] Public CDF note 9448; public DØ note 5715-CONF.

[5] T. Aaltonen et al. (CDF Collaboration), submitted to Phys. Rev. Lett., arXiv:0807.4262; T. Aaltonen et al. (CDF Collaboration), Phys. Rev. D78,111101 (2008); public CDF note 9432;

[6] V.M. Abazov et al. (DØ Collaboration), Phys. Rev. Lett. 100, 142002 (2008).

[7] T. Aaltonen et al. (CDF Collaboration), Phys. Rev. Lett. 101, 202001 (2008).

[8] V.M. Abazov et al. (DØ Collaboration), Phys. Rev. Lett. 101, 221801 (2008).

[9] Public DØ note 5722-CONF; T. Aaltonen et al. (CDF Collaboration), submitted to Phys. Lett. B, arXiv:0811.0344.

[10] Tevatron Electroweak Working Group and CDF and DØ Collaborations, arXiv:0808.1089.

[11] Public CDF note 9427; public DØ note 5750-CONF.

[12] Public CDF note 9157; public CDF note 9164; public DØ note 5600-CONF.

[13] V.M. Abazov et al. (DØ Collaboration), submitted to Phys. Rev. Lett., arXiv:0807.0859; V.M. Abazov et al. (DØ Collaboration), Phys. Rev. Lett. 100, 211803 (2008); public CDF note 9150.

[14] Public CDF note 9322; public DØ note 5715-CONF

[15] T. Aaltonen et al. (CDF Collaboration), Phys. Rev. Lett. 101, 192002 (2008).

[16] T. Aaltonen et al. (CDF Collaboration), submitted to Phys. Rev. Lett., arXiv:0812.3400.

[17] Public CDF note 9446.

[18] Public CDF note 9439. 